\title{
Masses and decay constants of (axial-)vector mesons at finite chemical potential
}

\author{
Pascal J. Gunkel ${ }^{1, \mathrm{a}} \mathbb{D}$, Christian S. Fischer ${ }^{1,2, \mathrm{~b}}{ }_{\mathbb{C}}$ \\ ${ }^{1}$ Institut für Theoretische Physik, Justus-Liebig-Universität Gießen, 35392 Gießen, Germany \\ ${ }^{2}$ Helmholtz Forschungsakademie Hessen für FAIR (HFHF), GSI Helmholtzzentrum für Schwerionenforschung, Campus Gießen, 35392 Gießen, \\ Germany
}

Received: 9 December 2020 / Accepted: 25 March 2021 / Published online: 24 April 2021

(C) The Author(s) 2021

Communicated by Ralf Rapp

\begin{abstract}
We update our previous results for (pseudo)scalar mesons at zero temperature and finite quark chemical potential and generalize the investigation to include (axial)vector mesons. We determine bound-state properties such as meson masses and decay constants up to chemical potentials far in the first-order coexistence region. To extract the bound-states properties, we solve the Bethe-Salpeter equation and utilize Landau-gauge quark and gluon propagators obtained from a coupled set of (truncated) Dyson-Schwinger equations with $N_{\mathrm{f}}=2+1$ dynamical quark flavors at finite chemical potential and vanishing temperature. For multiple (pseudo-)scalar and (axial-)vector mesons, we observe constant masses and decay constants for chemical potentials up to the coexistence region of the first-order phase transition thus verifying explicitly the Silver-Blaze property of QCD. Inside the coexistence region the pion becomes more massive and its decay constants decrease, whereas corresponding quantities for the (axial-)vector mesons remain (almost) constant.
\end{abstract}

\section{Introduction}

In the analysis of experimental heavy-ion collisions, electromagnetic radiation from the hot and dense fireball plays a pivotal role. Once the real or virtual photon is produced in the reaction, it escapes the medium almost undistorted and can therefore serve as a probe for the state of matter in the early stages of the collision. Due to their quantum numbers, vector mesons couple to the electromagnetic current and therefore in particular the light ones, $\rho, \omega$, and $\phi$ are expected to contribute substantially to the observed dilepton

\footnotetext{
${ }^{a}$ e-mail: pascal.gunkel@physik.uni-giessen.de (corresponding author)

b e-mail: christian.fischer@theo.physik.uni-giessen.de
}

spectrum. The study of the in-medium properties of vector mesons has thus received considerable attention, see, e.g., Refs. [1-3] for reviews.

The region of the QCD phase diagram with low temperatures and large densities is the realm of cold nuclear matter. The properties of vector mesons, in particular their spectral functions have been studied in a range of approaches with focus on the medium effects of their pion cloud as well as medium effects due to the coupling of the $\rho$ meson to nucleons via resonance excitations [1-3]. A very recent study in this direction takes into account quantum fluctuations via the functional renormalization group approach to a low-energy effective theory $[4,5]$.

Less studied is the direct impact of non-vanishing chemical potential on the quark and gluon structure of the $\rho$, its chiral partner $a_{1}$ and the $\phi$ meson and the resulting changes in their mass and decay constants. This is the topic of this work. Based on previous results for the chemical-potential dependence of the quark propagator and the resulting behavior of the masses and decay constants of pseudo-scalar and scalar mesons at finite chemical potential [6] we improve the approach and generalize it to also accommodate for vector and axial-vector mesons. Working at zero temperature, our study is complementary to a recent study of the thermal properties of vector mesons at and around the crossover at finite temperature [7].

One of the most interesting questions associated with the zero temperature, finite chemical potential axis of the QCD phase diagram is the Silver-Blaze property of QCD: For baryon chemical potentials smaller than the mass of the nucleon minus its binding energy in nuclear matter, there can be no excitations from the QCD vacuum and therefore all observables have to retain their vacuum values. This can be shown analytically for the case of finite isospin chemical potential, but is also extremely plausible for the case of 
finite baryon chemical potential [8,9] and has been demonstrated for heavy quark masses in the lattice formulation of Ref. [10]. How this works in the case of pseudoscalar and scalar mesons has been studied in Ref. [6]. Here, we will see that a similar mechanism is in place for the vector and axial-vector mesons.

The paper is organized as follows: In Sect. 2 we discuss the truncation of Dyson-Schwinger and Bethe-Salpeter equations that we use in our study. More details can be found in Ref. [6]. In Sect. 3 we present our updated results for the chemical-potential dependence of masses and decay constants of pseudo-scalar and scalar mesons as well as results for the vector and axial-vector mesons. We conclude in Sect. 4.

\section{Bethe-Salpeter formalism}

The homogeneous Bethe-Salpeter equation (BSE) for (pseudo-)scalar and (axial-)vector mesons in ladder truncation is given by

$$
\begin{aligned}
\Gamma_{\mathrm{x}, f}^{(\mu)}(p, P) & =-\left(Z_{2}^{f}\right)^{2} g^{2} C_{\mathrm{F}} \int_{q} \gamma_{\mu} S_{f}\left(q_{+}\right) \Gamma_{\mathrm{x}, f}^{(\mu)}(q, P) \\
& \times S_{f}\left(q_{-}\right) \gamma_{\nu} \Gamma\left(k^{2}\right) D_{\mu \nu}(k)
\end{aligned}
$$

with the shorthand $\int_{q} \equiv \int \mathrm{d}^{4} q /(2 \pi)^{4}$ and the strong coupling constant $g^{2}=4 \pi \alpha_{\mathrm{s}}$. The Casimir $C_{\mathrm{F}}=\left(N_{\mathrm{c}}^{2}-\right.$ 1) $/\left(2 N_{\mathrm{c}}\right)$ results from the color trace with $N_{\mathrm{c}}=3 . Z_{2}^{f}$ represents the quark wave function renormalization constant of the quark flavour $f \in\{\mathrm{u}, \mathrm{d}, \mathrm{s}\}$. The relative momenta of the meson $(p$ and $q)$ entail the quark chemical potential ${ }^{1} \mu_{\mathrm{q}}^{f}$ and are given by $p=\left(\vec{p}, \tilde{p}_{4}\right)$ with $\tilde{p}_{4}=p_{4}+i \mu_{\mathrm{q}}^{f}$. We consider the meson to be in its rest frame, i.e., $P=\left(\overrightarrow{0}, i m_{\mathrm{x}}\right)$ with the time-like total momentum $P$ and the mass $m_{\mathrm{x}}$ of the meson. The index $\mathrm{x}$ thereby describes the meson type. We use the momentum routings $k=p-q$ and $q_{ \pm}=q \pm \eta_{ \pm} P$ for the gluon and the quark momenta, respectively. The momentumpartitioning parameters $0 \leq \eta_{ \pm} \leq 1$ can be varied within the boundary condition $\eta_{+}+\eta_{-}=1$. In the vacuum, Poincaré covariance implies the independence of all observables on the choice of $\eta_{ \pm}$. Numerically, this is satisfied on the permille level. At finite chemical potential and for pseudoscalar mesons we explicitly verified that this invariance holds for chemical potentials up and into the coexistence region. For larger chemical potentials and for heavier mesons $\eta_{+}$needs to be adapted such that the integration in the BSE avoids the complex plane singularities in the quark propagators.

\footnotetext{
${ }^{1}$ In this work, we use a vanishing isospin $\mu_{\mathrm{I}}$ and strangeness $\mu_{\mathrm{S}}$ chemical potential implying $\mu_{\mathrm{q}}^{\mathrm{u}}=\mu_{\mathrm{q}}^{\mathrm{d}}=\mu_{\mathrm{q}}^{\ell}$ and $\mu_{\mathrm{q}}^{\mathrm{s}}=\mu_{\mathrm{q}}^{\ell}$. Furthermore, we often express the chemical potential by the baryon chemical potential $\mu_{\mathrm{B}}=3 \mu_{\mathrm{q}}^{\ell}$
}

The homogeneous BSE depends on the dressed quark and gluon propagators $S_{f}$ and $D_{\mu v}$ as well as the dressed quarkgluon vertex with dressing function $\Gamma\left(k^{2}\right)$. A detailed discussion of our truncation for the vertex as well as explicit expressions for $\Gamma\left(k^{2}\right)$ and the corresponding choice of the coupling $\alpha_{\mathrm{s}}$ can be found in Ref. [6]. For finite quark chemical potential $\mu_{\mathrm{q}}^{f}$ and vanishing temperature, the Landau-gauge propagators can be written as

$$
\begin{aligned}
& S_{f}^{-1}(p)=i \overrightarrow{\not p} A_{f}(p)+i \tilde{p}_{4} \gamma_{4} C_{f}(p)+B_{f}(p), \\
& D_{\mu \nu}(k)=\mathcal{P}_{\mu \nu}^{\mathrm{T}}(k) \frac{Z_{\mathrm{T}}(k)}{k^{2}}+\mathcal{P}_{\mu \nu}^{\mathrm{L}}(k) \frac{Z_{\mathrm{L}}(k)}{k^{2}} .
\end{aligned}
$$

Here, the four-dimensional transverse projector is split into a part $\mathcal{P}_{\mu \nu}^{\mathrm{T}}(k)$ transverse to the assigned direction $v=(\overrightarrow{0}, 1)$ of the medium and a corresponding longitudinal part $\mathcal{P}_{\mu \nu}^{\mathrm{L}}(k)$. The associated dressing functions of the gluon split into the transverse (or magnetic) part $Z_{\mathrm{T}}$ and the longitudinal (or electric) part $Z_{\mathrm{L}}$. The gluon and quark $\left(A_{f}, B_{f}, C_{f}\right)$ dressing functions encode the non-trivial momentum dependence of the propagators. For vanishing chemical potential the vector dressing functions $A_{f}$ and $C_{f}$ as well as the gluon dressing functions degenerate. For finite chemical potential this is in general no longer the case.

The quark and gluon propagators are calculated from a coupled set of truncated Dyson-Schwinger equations. In the corresponding truncation, we use quenched lattice data for the gluon as input and unquench it explicitly by including the back-reaction of the quark onto the gluon. Furthermore, we use an ansatz for the quark-gluon vertex. Details for this wellstudied truncation can be found in Ref. [11] and the review Ref. [12]. In previous works this truncation was used for the quenched case $[13,14]$ as well as for different numbers of quark flavors [15-18]. Also supercolorconductivity has been studied using this truncation [19,20]. Two further approximations were made in the preceding work of Ref. [6]: (i) the chemical-potential dependence of the gluon is neglected and (ii) a slightly modified quark-gluon vertex ansatz in the quark DSE is used. We build upon this work and adopt these approximations.

In the vacuum BSE we use the same tensor-structure decomposition for pseudoscalar $(\mathrm{x}=\mathrm{P})$ and scalar $(\mathrm{x}=\mathrm{S})$ mesons as detailed in Ref. [6]. In the medium we extend the Bethe-Salpeter amplitude (BSA) to

$$
\begin{aligned}
\Gamma_{\mathrm{P}}(p, P)= & \gamma_{5}\left\{E_{\mathrm{P}}(p, P)-i \overrightarrow{p p} P \cdot p G_{\mathrm{P}}^{\mathrm{s}}(p, P)+\right. \\
& \left.-i \gamma_{4} I_{\mathrm{P}}(p, P)\right\} \\
\Gamma_{\mathrm{S}}(p, P)= & \mathbb{1}_{\mathrm{D}}\left\{E_{\mathrm{S}}(p, P)-i \vec{p} G_{\mathrm{S}}^{\mathrm{s}}(p, P)-i \gamma_{4} I_{\mathrm{S}}(p, P)\right\}
\end{aligned}
$$

including the additional structure $G_{\mathrm{x}}^{\mathrm{s}}$ as compared to Ref. [6]. Results from the vacuum suggest that this addition will not 
change the meson masses by much, but may be relevant for the decay constant [21]. We will see later, that this is indeed the case. The flavor dependence of the amplitude is suppressed in our notation. For the vector $(\mathrm{x}=\mathrm{V})$ and axialvector $(\mathrm{x}=\mathrm{A})$ in vacuum we work with the tensor decomposition detailed in previous works (see, e.g., Refs. [22-24]):

$$
\begin{aligned}
& \Gamma_{\mathrm{V}}^{\mu}(p, P)=i \gamma_{\top}^{\mu} F_{1 \mathrm{~V}}(p, P)+\gamma_{\top}^{\mu} \not p F_{2 \mathrm{~V}}(p, P) \\
& +\left(p_{\top}^{\mu} \mathbb{1}_{\mathrm{D}}-\gamma_{\top}^{\mu} \not p\right) P \cdot p F_{3 \mathrm{~V}}(p, P) \\
& +\left(i \gamma_{\top}^{\mu}[\not P, \not p]+2 i p_{\top}^{\mu} \not \boldsymbol{P}\right) F_{4 \mathrm{~V}}(p, P) \\
& +p_{\top}^{\mu} \mathbb{1}_{\mathrm{D}} F_{5 \mathrm{~V}}(p, P)+i p_{\top}^{\mu} \not \boldsymbol{P} P \cdot p F_{6 \mathrm{~V}}(p, P) \\
& -i p_{\top}^{\mu} \not p F_{7 \mathrm{~V}}(p, P)+p_{\top}^{\mu}[\not \boldsymbol{P}, \not p] F_{8 \mathrm{~V}}(p, P), \\
& \Gamma_{\mathrm{A}}^{\mu}(p, P)=\gamma_{5}\left\{i \gamma_{\top}^{\mu} F_{1 \mathrm{~A}}(p, P)+\gamma_{T}^{\mu} \not P P \cdot p F_{2 \mathrm{~A}}(p, P)\right. \\
& +\left(p_{\top}^{\mu} \mathbb{1}_{\mathrm{D}}-\gamma_{\top}^{\mu} \not p\right) F_{3 \mathrm{~A}}(p, P) \\
& +\left(i \gamma_{\top}^{\mu}[\not \boldsymbol{P}, \not p]+2 i p_{\top}^{\mu} \not \boldsymbol{P}\right) F_{4 \mathrm{~A}}(p, P) \\
& +p_{\top}^{\mu} \mathbb{1}_{\mathrm{D}} P \cdot p F_{5 \mathrm{~A}}(p, P)+i p_{\top}^{\mu} \not P P \cdot p F_{6 \mathrm{~A}}(p, P) \\
& \left.-i p_{\top}^{\mu} \not p F_{7 \mathrm{~A}}(p, P)+p_{\top}^{\mu}[\not \boldsymbol{P}, \not p] P \cdot p F_{8 \mathrm{~A}}(p, P)\right\} \text {. }
\end{aligned}
$$

The tensor decomposition is constructed such that the onshell (axial-)vector meson is transverse to its total momentum $P$. The subscript $\top$ indicates transversality of $w \in\{\gamma, p\}$ w.r.t. the total momentum, i.e., $w_{\top}^{\mu}=T_{\mu \nu}(P) w^{\nu}$.

In medium, the number of independent transverse tensor structures increases from eight to 24 thus inducing considerable numerical costs. Therefore we only consider two BSA components $F_{1 \mathrm{x}}$ and $F_{4 \mathrm{x}}$ for the qualitative study of this work. While $F_{1 \mathrm{x}}$ is the dominant BSA component of the vector meson, $F_{4 \mathrm{x}}$ is the correspondingly dominant one for axial-vector mesons. In medium the BSA components splits up into a spatial $F_{i s \mathrm{~V}}$ and a temporal $F_{i \mathrm{tV}}$ component implying two separate uncoupled BSEs to solve. The employed tensor decomposition in medium is

$$
\begin{aligned}
\Gamma_{\mathrm{V}}^{\mu}(p, P)= & i \gamma_{\mathrm{TT}}^{\mu} F_{1 \mathrm{sV}}(p, P) \\
& +\left(i \gamma_{\top \mathrm{T}}^{\mu}[\not P, \not p]+2 i p_{\mathrm{T}}^{\mu} \not P\right) F_{4 \mathrm{sV}}(p, P) \\
& +i \gamma_{\mathrm{T}}^{\mu} F_{1 \mathrm{tV}}(p, P)+2 i p_{\mathrm{T} \mathrm{L}}^{\mu} P_{4} \gamma_{4} F_{4 \mathrm{tV}}(p, P), \\
\Gamma_{\mathrm{A}}^{\mu}(p, P)= & \gamma_{5}\left\{i \gamma_{\top \mathrm{T}}^{\mu} F_{1 \mathrm{sA}}(p, P)\right. \\
& +\left(i \gamma_{\mathrm{T}}^{\mu}[\not P, \not p]+2 i p_{\mathrm{T}}^{\mu} \not P\right) F_{4 \mathrm{sA}}(p, P) \\
& \left.+i \gamma_{\mathrm{T} \mathrm{L}}^{\mu} F_{1 \mathrm{tA}}(p, P)+2 i p_{\mathrm{T}}^{\mu} P_{4} \gamma_{4} F_{4 \mathrm{tA}}(p, P)\right\}
\end{aligned}
$$

with $w_{T(\mathrm{~T} / \mathrm{L})}^{\mu}=\mathcal{P}_{\mu \nu}^{\mathrm{T} / \mathrm{L}}(P) w^{\nu}$ and $w \in\{\gamma, p\}$. So far the $\rho$ meson at finite temperature was investigated using only the dominant BSA component $F_{1 \mathrm{~V}}$ with an effective interaction (see, e.g., Refs. [25-27]).

All amplitudes of the (pseudo-)scalar and (axial-)vector mesons are normalized using the Nakanishi method [28] and serve as input into the calculation of the pseudo-scalar and (axial-)vector meson decay constants $f_{\mathrm{x}}$. In vacuum these are given by

$f_{\mathrm{x}}=\frac{N_{\mathrm{c}}}{i m_{\mathrm{x}}} \int_{q} \operatorname{Tr}_{\mathrm{D}}\left\{j_{\mathrm{x}, f}^{(\mu)}(P) S_{f}\left(q_{+}\right) \hat{\Gamma}_{\mathrm{x}, f}^{(\mu)}(q, P) S_{f}\left(q_{-}\right)\right\}$,

where $\hat{\Gamma}_{\mathrm{x}, f}^{(\mu)}$ represents the normalized BSA and the current $j_{\mathrm{x}, f}^{(\mu)}(P)$ is defined by

$j_{\mathrm{x}, f}^{(\mu)}(P)= \begin{cases}Z_{2}^{f} \gamma_{5} \hat{p} & \text { for } \mathrm{x}=\mathrm{P} \\ Z_{2}^{f} \gamma_{T}^{\mu} \frac{1}{3} & \text { for } \mathrm{x}=\mathrm{V} . \\ Z_{2}^{f} \gamma_{5} \gamma_{T}^{\mu} \frac{1}{3} & \text { for } \mathrm{x}=\mathrm{A}\end{cases}$

Equation (8) is evaluated for the on-shell momentum $P^{2}=$ $-m_{\mathrm{x}}^{2}$ and is exact if the dressed quark propagators and the meson BSA are exact. The factor $1 / 3$ in the (axial-)vector case has to be included because of the summation over the polarizations.

In medium the decay constant of the pseudo-scalar meson splits into two parts $[29,30]$ as discussed in our previous work [6]. One arrives at

$f_{\mathrm{P}}^{\mathrm{s} / \mathrm{t}}=N_{\mathrm{c}} \int_{q} \operatorname{Tr}_{\mathrm{D}}\left\{j_{\mathrm{P}, f}^{\mathrm{s} / \mathrm{t}}(P) S_{f}\left(q_{+}\right) \hat{\Gamma}_{\mathrm{P}, f}^{\mu}(q, P) S_{f}\left(q_{-}\right)\right\}$,

with the corresponding current

$j_{\mathrm{P}, f}^{\mathrm{s} / \mathrm{t}}(P)=\left\{\begin{array}{ll}Z_{2}^{f} \gamma_{5} \overrightarrow{\boldsymbol{P}} / \vec{P}^{2} & \text { for } \mathrm{s} \\ Z_{2}^{f} \gamma_{5} \gamma_{4} / P_{4} & \text { for } \mathrm{t}\end{array}\right.$.

For the (axial-)vector meson in medium we can equally define a spatial and temporal decay constant belonging to the spatial and temporal BSA, respectively:

$$
f_{\mathrm{x}}^{\mathrm{s} / \mathrm{t}}=\frac{N_{\mathrm{c}}}{i m_{x}} \int_{q} \operatorname{Tr}_{\mathrm{D}}\left\{j_{\mathrm{x}, f}^{\mathrm{s} / \mathrm{t}, \mu}(P) S_{f}\left(q_{+}\right) \hat{\Gamma}_{\mathrm{x}, f}^{\mu}(q, P) S_{f}\left(q_{-}\right)\right\}
$$

In this equation the current for vector mesons is defined as

$j_{\mathrm{V}, f}^{\mathrm{s} / \mathrm{t}, \mu}(P)=\left\{\begin{array}{ll}Z_{2}^{f} \gamma_{T \mathrm{~T}}^{\mu} \frac{1}{2} & \text { for } \mathrm{s} \\ Z_{2}^{f} \gamma_{T \mathrm{~L}}^{\mu} & \text { for } \mathrm{t}\end{array}\right.$.

In case of axial-vector mesons a $\gamma_{5}$ factor has to be included in the the current.

\section{Results}

In this section, we discuss our results for the masses and decay constants of light and strange (pseudo)-scalar and (axial- 
)vector mesons at non-vanishing chemical potential. In the discussion, we denote by 'coexistence region' the region of $\mu_{B}$ where both, the chirally-broken Nambu solution and the chirally symmetric Wigner solution are available and attractive in the iteration process. The 'spinodal points' are the end points of this region. For smaller chemical potentials the Wigner solution is still present (see e.g. [31]) but is not iteratively attractive. For larger chemical potentials the Nambu solution ceases to exist.

Note that in principle it is possible to determine the thermodynamic potential in our approach and therefore determine the boundary of first order transition within the coexistence region. However, for the coupled system of DSEs that we use in this work this is a non-trivial numerical task that requires considerable additional effort. Since thermodynamics is not the main issues of this work we postponed this task to a future work.

All our results are calculated using the Nambu solution of the quark DSE. The Wigner solution features poles close to the time-like real momentum axis at very low masses, posing technical problems in the Bethe-Salpeter equation which are beyond current solution techniques.

In Ref. [6] we studied the areas of stability of the Nambu and Wigner solution for the light quark at finite (light) chemical potential and vanishing temperature and located the coexistence region where both solutions exist and are stable. Here, in contrast to the previous work we additionally use a non-vanishing strange-quark chemical potential and investigate the simplest case $\mu_{\mathrm{q}}^{\mathrm{u}}=\mu_{\mathrm{q}}^{\mathrm{d}}=\mu_{\mathrm{q}}^{\mathrm{s}}$. In the fully back-coupled system, the first order transition of the up/down quark sector at some critical chemical potential then necessarily introduces non-analytic changes also in the strange quark propagator (see e.g. [32] for an explicit calculation of this effect). However, it is not clear whether the loss in interaction strength due to almost massless (and screening) up/down quarks is sufficient to reduce the strange quark immediately to its Wigner solution. Instead it might be that within some region of chemical potential the strange quark still feels dynamical chiral symmetry breaking (however with reduced strength) whereas the up/down sector is already in the Wigner mode. Then at even larger chemical potential a second first order transition in the strange quark sector would occur. Whether this scenario is realistic is an open question that remains to be studied.

In this work, however, we neglect the chemical-potential dependency of the gluon by always using the (unquenched) gluon propagator from the vacuum. ${ }^{2}$ Thus, any changes in the light and strange quark sector induced by chemical potential are not back-coupled to the respective other sector. Therefore, we do expect to find two different coexistence areas

\footnotetext{
$\overline{2}$ In Ref. [33] the influence of this approximation was studied for the case of two color QCD.
}

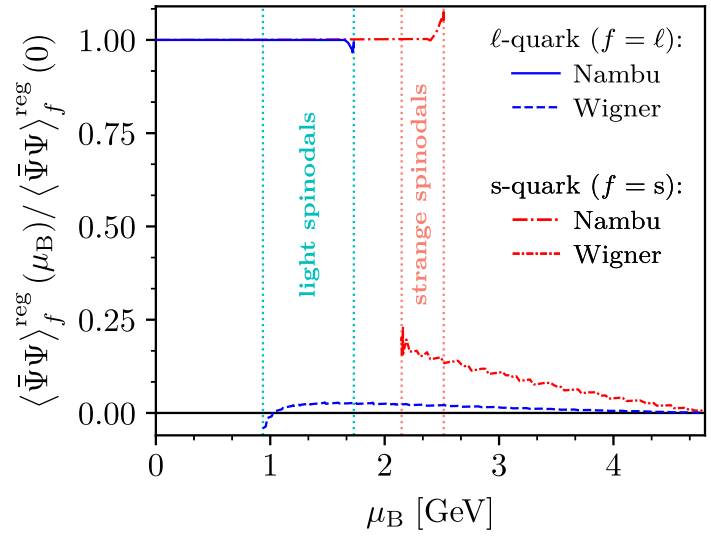

Fig. 1 Vacuum normalized and regularized light (blue) and strange (red) quark condensate for the chirally-broken Nambu (solid and dashed lines) and chirally-restored Wigner (differently dashed dotted lines) solution plotted against the baryon chemical potential $\mu_{\mathrm{B}}$. The boundaries for the appearance/disappearance of the Nambu and Wigner solution are denoted by vertical dotted lines in the corresponding color of the flavor and called light and strange spinodals

for the light and strange quark. Indeed, this can be seen in Fig. 1, where we show our results for the vacuum normalized and regularized ${ }^{3}$ light $\langle\bar{\Psi} \Psi\rangle_{\ell}$ and strange $\langle\bar{\Psi} \Psi\rangle_{\mathrm{s}}$ quark condensate plotted against the baryon chemical potential $\mu_{\mathrm{B}}=3 \mu_{\mathrm{q}}^{\ell}=3 \mu_{\mathrm{q}}^{\mathrm{s}}$. We also plot the boundaries of the coexistence regions to guide the eye. We find these at:

Wigner: Nambu:

$\mu_{B}=0.936 \mathrm{GeV}, 1.730 \mathrm{GeV}$ (light),

$\mu_{B}=2.149 \mathrm{GeV}, 2.516 \mathrm{GeV}$ (strange)

Slight changes as compared to Ref. [6] are due to improved numerics.

\subsection{Chemical-potential dependence of the meson properties}

In Fig. 2 we display the pion mass and temporal decay constant in vacuum and at finite chemical potential for two different levels of approximation of the Bethe-Salpeter amplitude (BSA). We show results from the most elaborated BSA truncation of Ref. [6] (dashed line, black) and the improved truncation used in this work (solid line, red). A corresponding colored symbol displays the results of the vacuum calculation. For our improved truncation this limit is smooth and well defined, whereas it is ambiguous for the truncation used in Ref. [6]. Therefore no symbol is shown for this case. It furthermore turns out that the addition of $G^{\mathrm{s}}$ only has a small effect for the pion mass, but has a significant quantitative

\footnotetext{
${ }^{3}$ We regularize the quark condensate by subtracting the quark condensate at very high chemical potential, where the dynamical part is expected to vanish: $\langle\bar{\Psi} \Psi\rangle_{f}^{\mathrm{reg}}\left(\mu_{\mathrm{B}}\right)=\langle\bar{\Psi} \Psi\rangle_{f}\left(\mu_{\mathrm{B}}\right)-\langle\bar{\Psi} \Psi\rangle_{f}(\infty)$.
} 


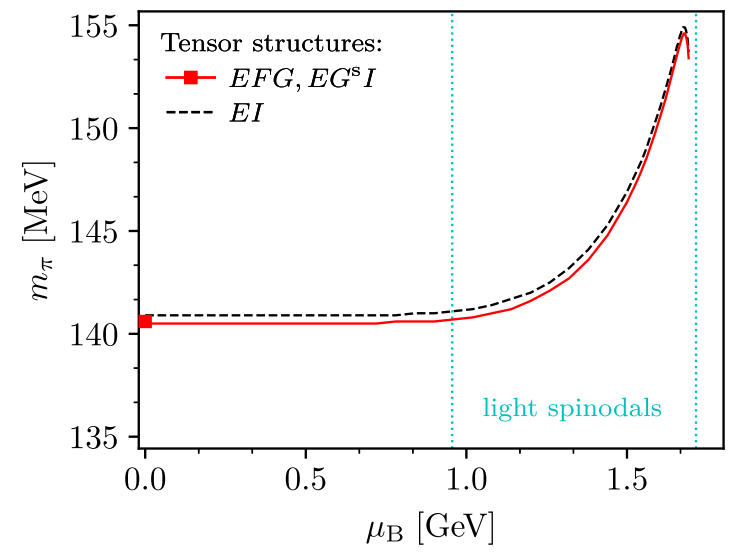

Fig. 2 Pion mass (left) and temporal pion decay constant (right) against the baryon chemical potential for different combinations of tensor structures used in the BSE calculation. The colored symbols

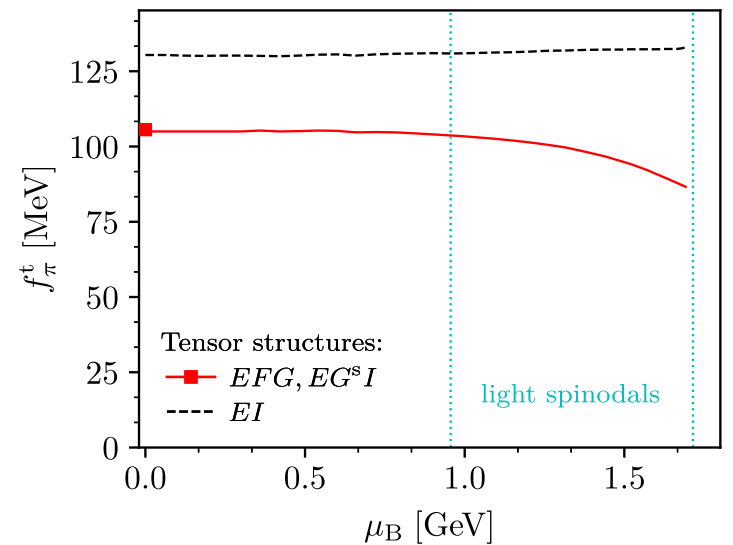

represent the corresponding vacuum results. All results are obtained with the chirally-broken Nambu solution. The vertical lines indicate the boundaries of the light quark coexistence region
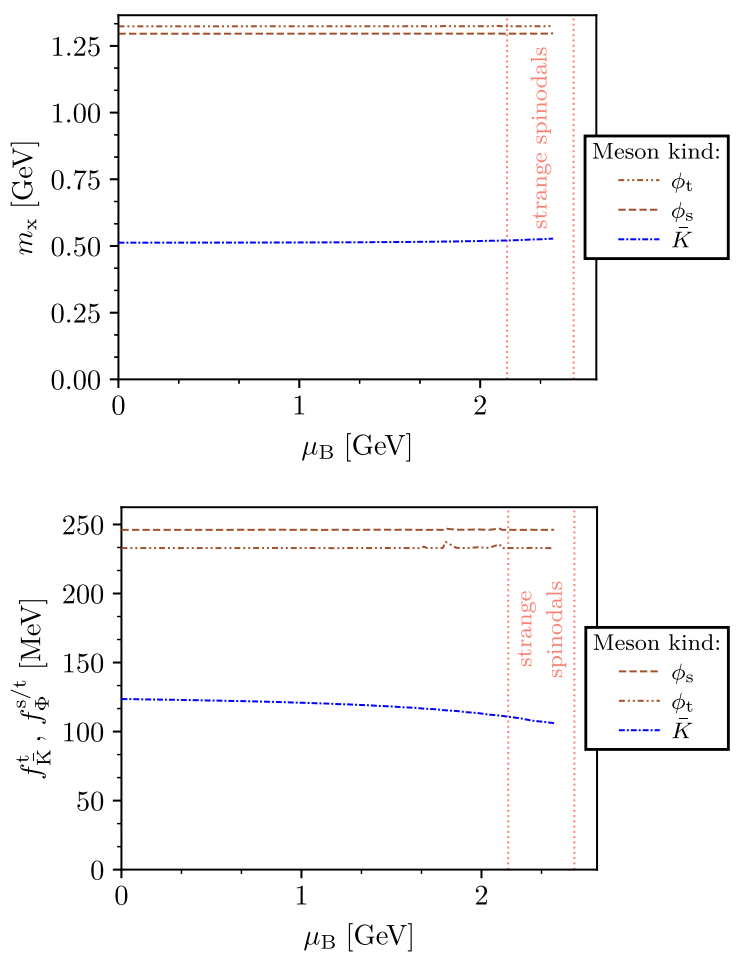

Fig. 3 Masses (upper) and decay constants (lower) for different light quark (left) and strange quark (right) mesons plotted against the baryon chemical potential for the most elaborated tensor structure combination of the BSE in medium. The results are calculated using the chirally-broken Nambu solution

effect of the order of $20 \%$ on the temporal decay constant, as anticipated above.

Within the numerical precision the pion mass and temporal decay constant remain constant up to a baryon chemical potential equal to the mass of a nucleon $m_{\mathrm{N}}=0.928 \mathrm{GeV}$ in medium. In this region, the mass and decay constant deviate by less than $0.25 \%$ and $2 \%$ from their vacuum values. This is constant within numerical accuracy. Consequently, we can state that the pion properties fulfill the Silver-Blaze prop- erty. Until the end of the coexistence region the pion mass increases up to $14 \%$ compared to the vacuum value. For the temporal pion decay constant from our improved truncation scheme we find a $20 \%$ decrease compared to the vacuum value.

In Fig. 3 we show the meson properties for multiple light and strange quark mesons at finite chemical potential in our improved truncation scheme. The $K$ and $\bar{K}$ meson behave similarly as the $\pi$ meson. But while the observed decrease of 
the decay constant is less pronounced for the $K$ meson, the contrary is true for the $\bar{K}$ meson. Overall the kaon masses increase by less than $3 \%$ while the decay constant decrease by $15 \%$. For the $\sigma$ meson there is no significant qualitative or quantitative difference between the different levels of approximation of the BSA as used in Ref. [6] and here (therefore we only display the updated result). The mass of the $\sigma$-meson and the longitudinal and transversal $\rho$ and $\phi$ properties remain perfectly constant until the end of the corresponding coexistence area with a maximal deviation of less than $0.5 \%$. Again we note that the Silver-Blaze property is very well satisfied.

The masses and decay constant of the temporal and spatial projections of the $\rho$ and $\phi$ meson have different values and we find that only the spatial quantities have a smooth vacuum limit. The reason is that the temporal projections of the BSA at finite chemical potential receive contributions from several vacuum BSA components and therefore the vacuum limit becomes ambiguous. The inclusion of the subleading second tensor structure $F_{4}$ reduces the difference between the properties of the spatial and longitudinal projection of the vector mesons drastically. Due to numerical reasons only the spatial projection of the $\mathrm{a}_{1}$ meson can be calculated. The mass of the spatial projection of the $a_{1}$ meson remains perfectly constant until the end of the light-quark coexistence region.

We wish to point out that previous works in the DSE/BSE framework also find meson properties at finite chemical potential which satisfy the Silver-Blaze property approximately [26,34-37]. These works use effective interactions and a number of further approximations for the bound-state calculation. Nevertheless, qualitatively they deliver similar results than our approach at least up to the coexistence region.

It should be noted that the constant behaviour of masses and decay constants in the Silver-Blaze region up to a baryon chemical potential of the mass of the nucleon minus its binding energy in nuclear matter is a highly non-trivial matter that relies on subtle cancellations between the chemicalpotential dependence of the quarks, their interaction inside the mesons and the Bethe-Salpeter amplitudes (to be discussed below), which together conspire to produce constant masses and decay constants. To our mind, it is very satisfying to find that this property holds in the functional approach.

\subsection{Bethe-Salpeter amplitudes}

We discussed the chemical-potential dependency of the $\pi$ and $\sigma$ meson BSAs in detail already in Ref. [6]. Here we reconsider these briefly and discuss updates due to our improved truncation scheme and detail in addition the corresponding behaviour of the BSAs of the strange (pseudo-)scalars and the (axial-)vector mesons included in this work. A general property of all BS amplitudes for non-vanishing chemical potential is that they develop an imaginary part and they loose their symmetry properties under charge conjugation [6].

In Fig. 4, we display the real part of all three mediumBSA components of the $\pi$ meson for fixed $P^{2}=-m_{\pi}^{2}$ plotted against the relative momentum $p$ between the quarks for different chemical potentials. For a given chemical potential we furthermore plot amplitudes with different angles $\hat{p} \hat{P}$ between total and relative momentum. The spread of the different line types is therefore a direct measure for the angular dependence of the amplitudes. All three components show a similar behavior: With increasing chemical potential all become larger in the infrared, they all spread more in the mid-momentum region and do not react to chemical potential in the ultraviolet momentum region. The strength of the infrared increase, however, is different for the different BSA components. While $G^{\mathrm{s}}$ almost doubles its magnitude and is therefore comparable in strength to the leading BSA component $E$ at large chemical potential, $I$ increases only weakly. Together this underlines the importance of taking $G^{\mathrm{s}}$ into account, especially at large chemical potential. For small chemical potentials all BSA components connect smoothly to the vacuum limit.

The $K$ meson BSAs (not shown) behave qualitatively similar as the $\pi$ BSA's but the changes are much less pronounced. Most importantly, $G^{\mathrm{s}}$ remains weak and does not become comparable to the $E$ component. For the $\bar{K}$ meson $G^{\mathrm{s}}$ even decreases whereas $I$ shows a stronger increase compared to the $K$ meson. The $I$ - and $G^{\mathrm{s}}$ - BSA components of the $\sigma$ meson decrease marginally in the infrared and increase their spread in the mid-momentum region for increasing chemical potentials. All other mesons BSA's and in particular those for the (axial-)vector mesons show a very weak chemical-potential dependence in their real part, but a significant dependence in their imaginary part. This can be seen explicitly for the lading tensor component of the $\rho$ meson in the lower panel of Fig. 4. Thus, it is the imaginary part of the amplitude that balances the variations in the quark propagator with respect to chemical potential and therefore provides for Silver-Blaze property.

\section{Summary and conclusions}

In this work we have studied the masses and decay constants of light and strange (pseudo-)scalar and (axial-)vector mesons at finite chemical potential up to and into the coexistence region of the first order chiral phase transition. To this end we employed a coupled system of Dyson-Schwinger and Bethe-Salpeter equations for the unquenched gluon propagator and $N_{f}=2+1$ quark flavours in a truncation which has been discussed and probed already elsewhere [6].

For all meson types we find constant values for masses and spatial decay constants at least up to a baryon chemical 

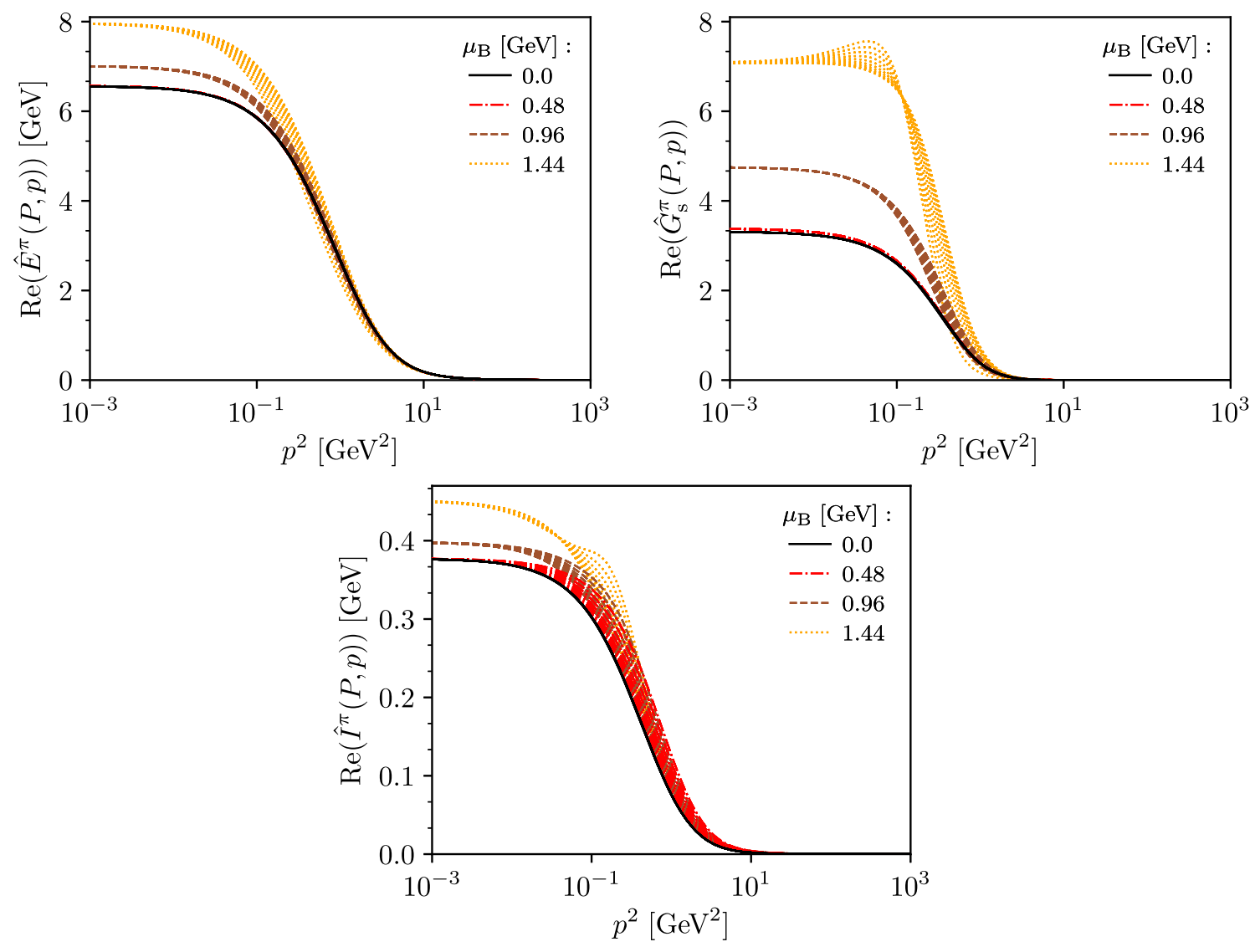

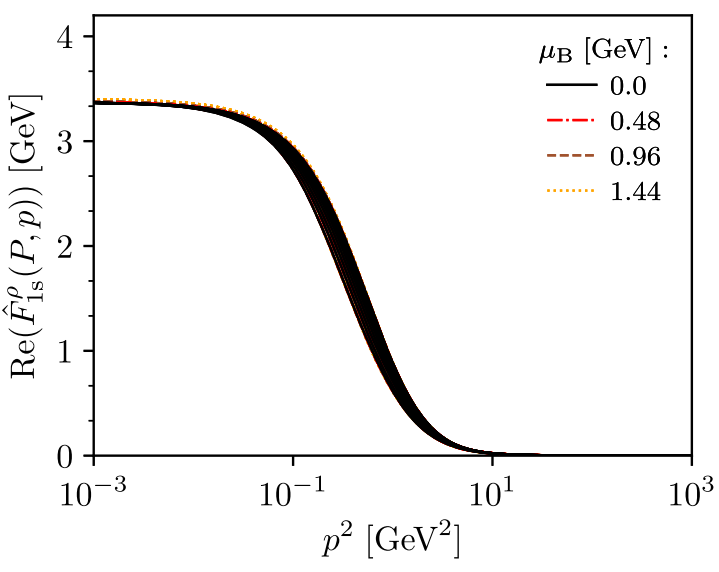

Fig. 4 Upper panel: Real part of the first and second normalized onshell medium $\pi$-BSA component $\hat{E}^{\pi}$ (left) and $\hat{G}_{\mathrm{s}}^{\pi}$ (right) ploted against the relative momentum $p^{2}$ between the quark and the antiquark for various baryon chemical potentials $\mu_{\mathrm{B}}$ far into the coexistence region. The spread of the amplitude results from the dependence on the angle between $P$ and $p$. The results are calculated for the chirally-broken

potential equal to the mass of the nucleon minus its binding energy in nuclear matter. Thus the Silver-Blaze property of QCD $[8,9]$ is at work. Since all input ingredients into the Bethe-Salpeter equation describing these mesons do depend on chemical potential, it is a highly non-trivial matter that the meson's Bethe-Salpeter amplitudes adapt and conspire such

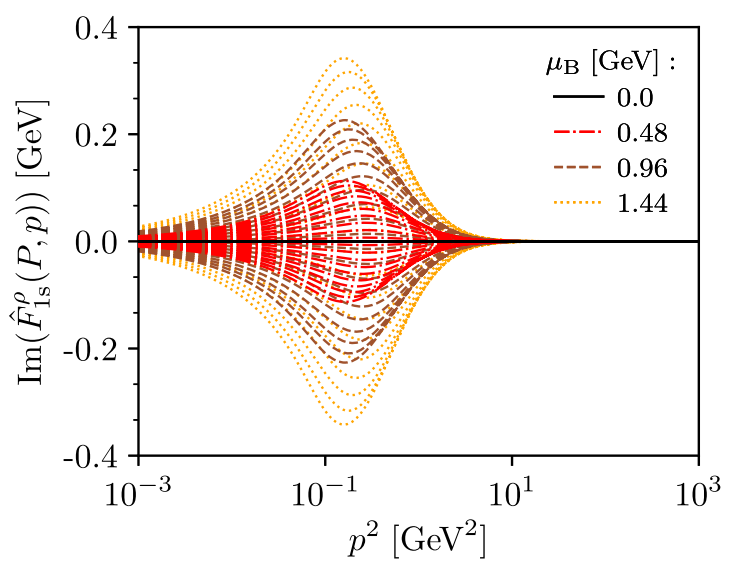

Nambu solution only. Middle panel: Real part of the third normalized on-shell medium $\pi$-BSA component $\hat{I}^{\pi}$. Lower panel: Real (left) and imaginary (right) part of the leading tensor component $\hat{F}_{1 s}^{\rho}$ of the rho meson. For the real part, the chemical potential dependence of the BSA is very weak, whereas much stronger variations are visible in the imaginary part

that observable quantities remain unaffected. To our mind, it is very satisfying to find that this property holds in the functional approach.

Acknowledgements We thank Richard Williams and Philipp Isserstedt for valuable discussions and Philipp Isserstedt for a careful reading 
of the manuscript. This work has been supported by the Helmholtz Graduate School for Hadron and Ion Research (HGS-HIRe) for FAIR, the GSI Helmholtzzentrum für Schwerionenforschung, and the BMBF under contract No. 05P18RGFCA.

Funding Information Open Access funding enabled and organized by Projekt DEAL.

Data Availability Statement This manuscript has associated data in a data repository. [Authors' comment: All data relevant for this project are shown in the plots and are available from the authors on demand.]

Open Access This article is licensed under a Creative Commons Attribution 4.0 International License, which permits use, sharing, adaptation, distribution and reproduction in any medium or format, as long as you give appropriate credit to the original author(s) and the source, provide a link to the Creative Commons licence, and indicate if changes were made. The images or other third party material in this article are included in the article's Creative Commons licence, unless indicated otherwise in a credit line to the material. If material is not included in the article's Creative Commons licence and your intended use is not permitted by statutory regulation or exceeds the permitted use, you will need to obtain permission directly from the copyright holder. To view a copy of this licence, visit http://creativecomm ons.org/licenses/by/4.0/.

\section{References}

1. R. Rapp, J. Wambach, Adv. Nucl. Phys. 25, 1 (2000). https://doi. org/10.1007/0-306-47101-9_1. arXiv:hep-ph/9909229 [hep-ph]

2. S. Leupold, V. Metag, U. Mosel, Int. J. Mod. Phys. E 19, 147 (2010). https://doi.org/10.1142/S0218301310014728. arXiv:0907.2388 [nucl-th]

3. B. Friman, C. Hohne, J. Knoll, S. Leupold, J. Randrup, R. Rapp, and P. Senger, eds., The CBM physics book: Compressed baryonic matter in laboratory experiments, Vol. 814 (2011) https://doi.org/ 10.1007/978-3-642-13293-3

4. C. Jung, F. Rennecke, R.-A. Tripolt, L. von Smekal, J. Wambach, Phys. Rev. D 95, 036020 (2017). https://doi.org/10. 1103/PhysRevD.95.036020. arXiv:1610.08754 [hep-ph]

5. C. Jung, L. von Smekal, Phys. Rev. D 100, 116009 (2019). https:// doi.org/10.1103/PhysRevD.100.116009. arXiv:1909.13712 [hep$\mathrm{ph}]$

6. P.J. Gunkel, C.S. Fischer, P. Isserstedt, Eur. Phys. J. A 55, 169 (2019). https://doi.org/10.1140/epja/i2019-12868-1. arXiv:1907.08110 [hep-ph]

7. L.-F. Chen, S.-X. Qin, Y.-X. Liu, (2020), arXiv:2006.10582 [hep$\mathrm{ph}]$

8. T.D. Cohen, R.J. Furnstahl, D.K. Griegel, Phys. Rev. C 45, 1881 (1992). https://doi.org/10.1103/PhysRevC.45.1881

9. T. D. Cohen, arXiv:hep-ph/0405043 (2004)

10. M. Fromm, J. Langelage, S. Lottini, M. Neuman, O. Philipsen, Phys. Rev. Lett. 110, 122001 (2013). https://doi.org/10.1103/ PhysRevLett.110.122001. arXiv:1207.3005 [hep-lat]

11. G. Eichmann, C.S. Fischer, C.A. Welzbacher, Phys. Rev. D 93, 034013 (2016). https://doi.org/10.1103/PhysRevD.93.034013. arXiv:1509.02082 [hep-ph]

12. C.S. Fischer, Prog. Part. Nucl. Phys. 105, 1 (2019). https://doi.org/ 10.1016/j.ppnp.2019.01.002. arXiv:1810.12938 [hep-ph]

13. C.S. Fischer, Phys. Rev. Lett. 103, 052003 (2009). https://doi.org/ 10.1103/PhysRevLett.103.052003. arXiv:0904.2700 [hep-ph]

14. C.S. Fischer, A. Maas, J.A. Mueller, Eur. Phys. J. C 68, 165 (2010). https://doi.org/10.1140/epjc/s10052-010-1343-1. arXiv:1003.1960 [hep-ph]
15. C.S. Fischer, J.A. Mueller, Phys. Rev. D 84, 054013 (2011). https:// doi.org/10.1103/PhysRevD.84.054013. arXiv:1106.2700 [hep-ph]

16. C.S. Fischer, J. Luecker, J.A. Mueller, Phys. Lett. B 702, 438 (2011). https://doi.org/10.1016/j.physletb.2011.07.039. arXiv:1104.1564 [hep-ph]

17. C.S. Fischer, J. Luecker, Phys. Lett. B 718, 1036 (2013). https://doi. org/10.1016/j.physletb.2012.11.054. arXiv:1206.5191 [hep-ph]

18. C.S. Fischer, J. Luecker, C.A. Welzbacher, Phys. Rev. D 90, 034022 (2014). https://doi.org/10.1103/PhysRevD.90.034022. arXiv: 1405.4762 [hep-ph]

19. D. Müller, M. Buballa, J. Wambach, Eur. Phys. J. A 49, 96 (2013). https://doi.org/10.1140/epja/i2013-13096-5. arXiv:1303.2693 [hep-ph]

20. D. Müller, M. Buballa, J. Wambach, arXiv:1603.02865 [hep-ph] (2016)

21. P. Maris, C.D. Roberts, P.C. Tandy, Phys. Lett. B 420, 267 (1998a). https://doi.org/10.1016/S0370-2693(97)01535-9. arXiv:nucl-th/9707003 [nucl-th]

22. C.L. Smith, Ann. Phys. 53, 521 (1969)

23. C.S. Fischer, P. Watson, W. Cassing, Phys. Rev. D 72, 094025 (2005). https://doi.org/10.1103/PhysRevD.72.094025. arXiv:hep-ph/0509213 [hep-ph]

24. R. Williams, EPJ Web Conf. 3, 03005 (2010). https://doi.org/10. 1051/epjconf/20100303005. arXiv:0912.3494 [hep-ph]

25. P. Maris, P. C. Tandy, in Research Program at the Erwin Schrödinger Institute on Confinement Vienna, Austria, May 5-July 17, 2000 (2001) arXiv:nucl-th/0109035 [nucl-th]

26. P. Maris, C.D. Roberts, S.M. Schmidt, Phys. Rev. C 57, R2821 (1998b). https://doi.org/10.1103/PhysRevC.57.R2821. arXiv:nucl-th/9801059 [nucl-th]

27. P. Maris, P.C. Tandy, Phys. Rev. C 60, 055214 (1999). https://doi. org/10.1103/PhysRevC.60.055214. arXiv:nucl-th/9905056 [nuclth]

28. N. Nakanishi, Phys. Rev. 138, B1182 (1965). https://doi.org/10. 1103/PhysRev.138.B1182

29. D.T. Son, M.A. Stephanov, Phys. Rev. Lett. 88, 202302 (2002a). https://doi.org/10.1103/PhysRevLett.88.202302. arXiv:hep-ph/0111100

30. D.T. Son, M.A. Stephanov, Phys. Rev. D 66, 076011 (2002b). $\quad$ https://doi.org/10.1103/PhysRevD.66.076011. arXiv:hep-ph/0204226

31. C.S. Fischer, D. Nickel, R. Williams, Eur. Phys. J. C 60, 47 (2009). https://doi.org/10.1140/epjc/s10052-008-0821-1. arXiv:0807.3486 [hep-ph]

32. K. Otto, M. Oertel, B.-J. Schaefer, Phys. Rev. D 101, 103021 (2020). https://doi.org/10.1103/PhysRevD.101.103021. arXiv:1910.11929 [hep-ph]

33. R. Contant, M.Q. Huber, Phys. Rev. D 101, 014016 (2020). https:// doi.org/10.1103/PhysRevD.101.014016. arXiv:1909.12796 [hep$\mathrm{ph}]$

34. A. Bender, G.I. Poulis, C.D. Roberts, S.M. Schmidt, A.W. Thomas, Phys. Lett. B 431, 263 (1998). https://doi.org/10.1016/ S0370-2693(98)00546-2. arXiv:nucl-th/9710069 [nucl-th]

35. Y.-X. Liu, J.-Y. Chao, L. Chang, W. Yuan, Chin. Phys. Lett. 22, 46 (2005). https://doi.org/10.1088/0256-307X/22/1/014

36. Y. Jiang, Y.-M. Shi, H. Li, W.-M. Sun, H.-S. Zong, Phys. Rev. D 78, 116005 (2008). https://doi.org/10.1103/PhysRevD.78.116005. arXiv:0810.0750 [nucl-th]

37. Y. Jiang, Y.-M. Shi, H.-T. Feng, W.-M. Sun, H.-S. Zong, Phys. Rev. C 78, 025214 (2008). https://doi.org/10.1103/PhysRevC.78. 025214 